\title{
Update in Hospice and Palliative Care
}

\author{
Karen Hyden, MSN/Ed, APRN-BC, ACHPN, ${ }^{1}$ Laura Gelfman, MD, MPH, ${ }^{2,3}$ \\ J. Nicholas Dionne-Odom, PhD, APRN, ${ }^{4}$ Cardinale B. Smith, MD, PhD, 2,5 \\ and Heather Coats, PhD, APRN-BC ${ }^{1}$
}

\begin{abstract}
The purpose of this update is to summarize and critique research articles in Hospice and Palliative Care from 2018 that are pertinent and impactful in informing clinical practice. The articles were presented at the 2019 Annual Assembly of Hospice and Palliative Medicine (AAHPM) and the Hospice and Palliative Nurses Association (HPNA). Eight original research articles published between January 1, 2018, and December 31, 2018, were identified through a systematic PubMed search using the terms "hospice" and "palliative care," a hand search of 22 leading healthcare journals, and discussion with experts in the field. The final articles were chosen based on each study's methodological quality, its ability to be applied across different care settings, and its ability to impact clinical practice. We summarize the eight articles that were chosen and identify ways the articles can be used to inform clinical practice.
\end{abstract}

Keywords: hospice clinical care; palliative clinical care; state of science; update in hospice; update in palliative care

\section{Introduction}

$\mathbf{T}$ HE PURPOSE OF THIS UPDATE, presented at the 2019 Annual Assembly of Hospice and Palliative Medicine (AAHPM) and Hospice and Palliative Nurses Association (HPNA) is to identify, summarize, and critique the eight research articles that were selected as most pertinent to clinical practice. The selection criteria were as follows: the quality of science, potential for impact, and appeal to a breadth of interest.

Eight reports of original research articles published between January 1, 2018, and December 31, 2018, were identified through a systematic PubMed search using the terms "hospice" and "palliative care," a hand search of 22 leading medical journals, and discussion with experts in the field. The articles were chosen and then ranked based on three criteria: methodological quality, application to variety of palliative care clinicians across different care settings, and the potential impact on clinical practice. We summarize the eight articles that were chosen based on the highest ratings and identify ways the articles can be used to inform clinical practice (Table 1).

\section{A Focus on Understanding Patients' Goals of Care \\ Summary and main findings: shared decision making in life-prolonging treatments}

Shared decision making between patient and clinicians is an important way to align treatments with the patients' values and wishes. As presented in the study, Effectiveness of an Intervention Supporting Shared Decision Making for Destination Therapy Left Ventricular Assist device: The DECIDE-LVAD Randomized Clinical Trail, shared decision making is especially important in the case of an invasive life-prolonging devices such as the left ventricular assist devices as destination therapy (DT-LVADs). These therapies are offered to patients with advanced heart failure who are not candidates for cardiac transplantation. ${ }^{1}$ Unfortunately, the processes in place to facilitate educational and shared decision-making conversations between patients and their clinicians are often not optimal. ${ }^{2}$ Decision aids have been shown to help facilitate successful shared decisionmaking conversations with patients who have a serious illness. $^{3}$

\footnotetext{
${ }^{1}$ College of Nursing, University of Colorado Denver, Anschutz Medical Campus, Aurora, Colorado.

${ }^{2}$ Brookdale Department of Geriatrics and Palliative Medicine, Icahn School of Medicine at Mount Sinai, New York, New York.

${ }^{3}$ Geriatric Research Education and Clinical Center, James J. Peters VA Medical Center, Bronx, New York.

${ }^{4}$ School of Nursing, University of Alabama at Birmingham (UAB), Birmingham, Alabama.

${ }^{5}$ Division of Hematology/Medical Oncology, Icahn School of Medicine at Mount Sinai, New York, New York.

Accepted September 19, 2019
} 
Table 1. Recommendations for Clinical Hospice and Palliative Care Practice Emerging From Selected Articles

\begin{tabular}{|c|c|}
\hline Consider & Reference \\
\hline $\begin{array}{l}\text { Employing a combined shared decision } \\
\text { making clinician training and patient } \\
\text { decision aid to assist patients who are } \\
\text { considering an LVAD }\end{array}$ & Allen et al. ${ }^{1}$ \\
\hline $\begin{array}{l}\text { Using the Jumpstart-Tips communication } \\
\text { primer to improve goals-of-care } \\
\text { documentation of patients with serious } \\
\text { illness in the outpatient setting }\end{array}$ & Curtis et al. ${ }^{4}$ \\
\hline $\begin{array}{l}\text { Training lay health workers to assist } \\
\text { patients with cancer in goals-of-care } \\
\text { discussions }\end{array}$ & Patel et al. ${ }^{7}$ \\
\hline $\begin{array}{l}\text { Integrating } P R I S M \text {, a skill-based } \\
\text { resilience training, into the care of } \\
\text { AYAs with cancer }\end{array}$ & $\begin{array}{l}\text { Rosenberg } \\
\text { et al. }\end{array}$ \\
\hline $\begin{array}{l}\text { Integrating a collaborative care approach } \\
\text { to improve depression and fatigue } \\
\text { for patients with heart failure }\end{array}$ & $\begin{array}{l}\text { Bekelman } \\
\text { et al. }{ }^{18}\end{array}$ \\
\hline $\begin{array}{l}\text { Assessing spousal needs from diagnosis } \\
\text { of their partner's illness through } \\
\text { bereavement }\end{array}$ & $\begin{array}{l}\text { Ornstein } \\
\text { et al. }^{25}\end{array}$ \\
\hline \multicolumn{2}{|l|}{ Start } \\
\hline $\begin{array}{l}\text { Adding olanzapine to standard } \\
\text { antiemetic regimens for patients } \\
\text { receiving chemotherapy }\end{array}$ & $\begin{array}{l}\text { Sutherland } \\
\text { et al. }\end{array}$ \\
\hline
\end{tabular}

AYAs, adolescents and young adults; PRISM, Promoting Resilience in Stress Management.

In the DECIDE-LVAD study, the authors conducted a randomized clinical trial to test whether a shared decision support intervention for patients considering DT-LVAD improved decision quality compared with usual care. The study was a multicenter cluster randomized, phased rollout, effectiveness clinical trial. Participants were 18 years or older and were eligible for a DT-LVAD. The study sample included 248 patients, 113 in the DT-LVAD intervention arm and 135 in the usual care arm. The participants in the intervention had usual care along with a 26-minute video, an 8page pamphlet decision aid, and a 2.5-hour clinician-directed decision support training session. Clinicians implemented the intervention via a 60-minute grand rounds on DT-LVAD decision making and a 90-minute coaching/demonstration session of materials. The participants in the usual care arm received a teaching session with a DT-LVAD coordinator, and did not receive coaching and demonstration of the materials. The primary outcome was the decision quality of the participants based on knowledge and values-choices concordance. Decision quality was measured before and immediately after formal education, at one month and six months. The secondary outcomes included decision conflict and decision regret, perceived stress, illness acceptance, depression, and quality of life.

The study showed that patient-reported treatment choice at one month favored DT-LVAD more in the control group (47 [59.5\% vs. 95 [91.3\%], $p=<0.001$ ). Though the correlation between stated values and patient-reported treatment choice at one month was stronger in the intervention group than in the control group, the study showed no improved correlation between stated values and actual treatment received by six months for the intervention compared with the control group (difference in Kendall's $\tau$, 95\% CI, -0.24 to 0.25 ). The adjusted rate of DT-LVAD implantation by six months was higher for participants in the control $(79.9 \%)$ group than those in the intervention group $(53.9 \%, p=0.008)$. This varied by site and there were no differences in decision conflict, regret, or preferred control.

\section{Strengths and limitations}

The study was robust using a large sample size and recruitment of participants from six diverse sites. The limitations to consider are that participants were mostly white men and there was missing data, mainly due to deaths of participants.

\section{Clinical bottom line}

A shared decision-making intervention for patients with heart failure who are making complex choices about DTLVAD modestly improved patient decision quality but did not improve the concordance between the patients' stated values and the treatment that was actually received. In addition, the rate of DT-LVAD implantation was substantially lower in the participants who were in the intervention arm compared to those who were in the usual care arm.

\section{Summary and Main Findings: Priming Interventions in Goals-of-Care Conversations}

According to focus groups including patients and caregivers, communication is mentioned more than any other palliative care intervention. ${ }^{4}$ However, it is still not fully understood whether interventions can help initiate and improve these conversations. ${ }^{5}$ In the article titled Effect of a Patient and Clinician Communication-Priming Intervention on PatientReported Goals-of-Care Discussions Between Patients with Serious Illness and Clinicians, it was demonstrated that clinician communication about goals of care has been associated with improved patient outcomes and reduced invasive end-oflife care. ${ }^{5}$

The communication-primer ( Jumpstart-Tips) intervention study, conducted between 2012 and 2016, was a clusterrandomized trial conducted in outpatient clinics. The clinicians ( $n=65$ in the intervention group; $n=67$ in the control group) were recruited from two large health care systems, which included two academic and two community hospitals, a comprehensive cancer center, and a substantial number of outpatient clinics. Eligible clinicians were physicians and nurse practitioners who were providing either primary or specialty care for patients with serious illnesses. Clinicians were randomized to the pre-conversation communicationpriming intervention $(n=65)$, or usual care $(n=67)$, and patients were assigned to either the intervention $(n=249)$ or usual care $(n=288)$ groups. ${ }^{6}$ The intervention was designed to increase goals-of-care conversations compared with usual care. The primary outcome was patient-reported occurrence of a goals-of-care conversation during a target outpatient visit. Secondary outcomes included clinician documentation of a goals-of-care conversation in the medical record, patientreported quality of communication measured by the Quality of Communication questionnaire ${ }^{6}$ at two weeks, patient 
assessments of goal-concordant care at three months, patientreported symptoms of depression measured by the 8 -item Patient Health Questionnaire (PHQ-8), and anxiety measured by the 7-item Generalized Anxiety Disorder survey (GAD-7) at three and six months.

The study showed that the Jumpstart-Tips intervention resulted in significant increase in number of patient-reported goals-of-care conversations during routine outpatient clinic visits $(74 \%$ vs. $31 \%, p=<0.001)$. Patients in the intervention group also reported increase in quality in the goals-of-care discussions (4.6 vs. $2.1, p=0.01$ ).

\section{Strengths and limitations}

This study is a high-quality multicenter randomized control trial. Limitations include potential geographical sample bias due to the study's conduct in a single region of the United States, and potential selection bias of participating patients and clinicians.

\section{Clinical bottom line}

A goals-of-care conversation priming intervention for patients with serious illness and outpatient clinicians may increase frequency and quality of goals-of-care conversations.

\section{Summary and Main Findings: Lay Health Workers and Goals-of-Care Conversations}

The delivery of high-quality end-of-life care for patients with cancer is challenging because few patients understand their prognoses, and many receive care, that is, different than their documented preferences. Communication between patients and clinicians about prognosis and end-of-life care may improve care and lower health care costs. ${ }^{7}$ Barriers such as time and reluctance on the part of the clinicians can prevent goals-of-care conversations from happening. ${ }^{7}$ The article titled, Effect of a Lay Health Worker Intervention on Goals-ofCare Documentation and on Health Care Use, Costs, and Satisfaction Among Patients with Cancer: A Randomized Clinical Trial, tested the use of training non-clinical personnel called lay health workers (LHW) as a way to fill the gap to improve patient's identification of their end-of-life care preferences. ${ }^{7}$ Lay health care workers have been integrated into the health care system since the mid-1960s, ${ }^{8}$ however, they are rarely integrated into end-of-life care, ${ }^{9,10}$ and there is limited available evidence on the effectiveness of integrating them into end-of-life care. ${ }^{11}$

In their study, the authors sought to determine whether an LHW program could increase documentation of patients' care preferences after a cancer diagnosis. The study was a randomized clinical trial with 213 patients who had diagnoses of stage 3 or 4 or recurrent cancer. The intervention was a six-month program with an LHW trained to assist patients with establishing end-of-life care preferences versus usual oncological care. The primary outcome was the documentation of goals of care. The secondary outcomes were patient satisfaction on the Consumer Assessment of Health Care Providers and Systems, consumer assessment of health care use, and costs. Results showed that the intervention significantly increased documentation of patients' end-of-life care preferences (97 [92.4\%] vs. 19 [17.5\%]; $p=<0.001)$ and improved patient satisfaction with their end-of-life-care decisions and the care they received $(p=<0.001)$.

\section{Strengths and limitations}

The study was a randomized controlled trial (RCT) design that offered a potentially highly scalable model of LHW-led support. Limitations to consider are that the study included a relatively small sample size, there was potential for geographical sample bias from using single VA site, there was a lack of attention control, and there was sampling bias toward white men.

\section{Clinical bottom line}

Adding an outpatient LHW program to usual care for patients with cancer has the potential to improve documentation of patients' goals of care at the end of life.

\section{A Focus on Adolescents and Young Adults}

\section{Summary and main findings: Resilience in adolescents and young adults with cancer}

Studies have shown that adolescents and young adults (AYAs), aged 12-25 years, with cancer are at risk for poor psychosocial outcomes. ${ }^{12}$ Cancer in the AYA population can result in impaired physical, social, and emotional health during and after cancer treatments. In addition, survivors often report greater psychological distress and fewer positive health beliefs than younger or older counterparts. ${ }^{12}$ Studies using adult participants with cancer have shown that techniques to promote resilience, such as goal setting, meaning making, and mindfulness, can result in decreased stress and distress, ${ }^{13}$ and improved immune function and overall health. ${ }^{14-16}$ In the article titled Promoting resilience in Adolescents and Young Adults With Cancer: Results from the PRISM Randomized Controlled Trial, the authors aimed to determine whether an intervention targeting resilience resources would improve patient-reported resilience, quality of life, and psychological distress.

The study was a phase II RCT. Ninety-two English-speaking AYAs with cancer were randomized to the Promoting Resilience in Stress Management ${ }^{12}$ (PRISM) intervention $(n=48)$ or usual care $(n=44)$. Participants completed surveys at enrollment and six months. Mixed effects regression models evaluated associations between PRISM and the primary outcome (10-item Connor-Davidson Resilience Scale scores) and secondary outcomes (generic and cancer-related quality of life [Pediatric Quality of Life modules], psychological distress [Kessler-6], and anxiety/depression [Hospital Anxiety and Depression]) at six months. The PRISM intervention was associated with improved resilience $(+3.0$ points; $95 \%$ CI, -4.1 to $-0.2, p=0.02)$, improved cancer-specific quality of life $(+9.6 ; 95 \% \mathrm{CI}, 2.6$ to $16.7, p=0.01)$, and reduced overall psychological distress in the adolescent patients $(-2.1$, $95 \% \mathrm{CI},-4.1$ to $-0.2, p=0.03)$. Generic quality of life was not improved $(+7.2 ; 95 \% \mathrm{CI},-0.8$ to $15.2, p=0.08)$.

\section{Strengths and limitations}

There was a high-quality RCT that tested a manualized and potentially highly scalable intervention using lay 
interventionists. Limitations to consider are that the study was conducted at a single site and included only Englishspeaking participants.

\section{Clinical bottom line}

PRISM, a brief skill-based intervention for AYAs, when added to usual care, is associated with better psychosocial outcomes, specifically resilience and cancer-specific quality of life and reduced psychological distress.

\section{A Focus on Interventions in Cancer Symptoms}

\section{Summary and main findings: Olanzapine for nausea and vomiting in cancer}

People with cancer often experience debilitating nausea and vomiting before, during, and after chemotherapy and/ or radiation treatments, despite accessing medications that are currently available to manage the distressing symptoms. The article titled Olanzapine for the Prevention and Treatment of Cancer-Related Nausea and Vomiting in Adults presents evidence that olanzapine may have the potential to help manage nausea and vomiting in this population. ${ }^{17}$ The authors sought to investigate the documented benefits and harms of using olanzapine for preventing and treating nausea and vomiting in adults with cancer.

The study was a Cochrane review of 14 RCTs of olanzapine versus any comparator with or without adjunct therapies for the prevention and/or treatment, of nausea or vomiting in 1917 participants across the studies, aged 18 or older, with cancer in any setting, of any duration, and with at least 10 participants per treatment arm. When olanzapine is added to standard therapy, it halves the likelihood of nausea and vomiting during chemotherapy from $50 \%$ to $25 \%$. However, the optimal dosage is unclear. The key findings of adverse events were that there was moderate quality evidence that the medication caused an increase in somnolence and fatigue, and there was low quality evidence of an increased risk of any serious adverse events.

\section{Strengths and limitations}

The strength of this article is a high-quality Cochrane Review using evidence from randomized controlled trails. However, the authors point out the RCTs included in their review had limited enrollments. Eight of the 14 RCTs had fewer than 50 participants in the treatment arm, and high risk of bias related to lack of blinding.

\section{Clinical bottom line}

In cancer patients, there is moderate quality evidence for the benefit of olanzapine when added to standard antiemetic regimens for the prevention and treatment of chemotherapyinduced nausea and vomiting. Yet, there is low quality evidence on adverse events and choice of optimal dose.

\section{A Focus on Collaborative Care}

\section{Summary and main findings: Collaborative versus usual care in chronic heart failure}

There are an estimated 5.8 million Americans living with debilitating symptoms such as reduced function and poor quality of life, caused by heart failure. These symptoms often result in a decreased overall health status. ${ }^{18}$ The National Academy of Medicine and the World Health Organization recommend palliative care for patients with heart failure ${ }^{19-21}$ because palliative care has been shown to improve the overall health status of these patients. ${ }^{20-22}$ However, there is a shortage of specialty - trained palliative care practitioners, and for the care to be optimally successful, it would need to be integrated into usual care earlier in the disease process. Ongoing care throughout the disease trajectory, and in the community-setting, could also increase the impact palliative care could have on the health status of patients with heart failure. $^{23}$ In the article, Effect of a Collaborative Care Intervention vs. Usual Care on Health Status of Patients with chronic Heart Failure: The CASA Randomized Clinical Trial, the authors investigated the Collaborative Care to Alleviate Symptoms and Adjust to Illness (CASA) intervention. ${ }^{18}$

The study was a controlled trial with 314 participants randomized to either an intervention $(n=157)$ or control $(n=175)$ arm. The intervention focused on symptoms and psychosocial collaborative care, in an effort to improve the patient-reported health status of patients with heart failure. The CASA intervention used a collaborative, health care delivery model to improve health status by addressing patient symptoms, adjustments to illness, and depression. The primary outcome was difference in heart failure-specific health status, measured using the Kansas City Cardiomyopathy Questionnaire at six months. Secondary outcomes included depression (7 item-Generalized Anxiety Disorder questionnaire), overall symptom distress (General Symptom Distress scale), specific symptoms of pain, fatigue, and shortness of breath, and number of hospitalizations and mortality. The mean score on the Kansas City Cardiomyopathy questionnaire did improve 5.5 points in intervention group and 2.9 points in control group (difference, 2.6 ; $95 \% \mathrm{CI},-1.3$ to 6.6 ) but this was not a significant change $(p=0.19)$. The secondary outcomes of depression and fatigue did improve with the intervention ( $p=0.02)$, but pain, shortness of breath, and overall symptom distress did not have any significant improvements. Mortality at 12 months was similar in both groups.

\section{Strengths and limitations}

The study was a multisite, RCT. The CASA intervention is scalable and easily integrated into usual outpatient care for this population. The limitations to consider are a lack of attention control or usual care measurement. Further, the sample was comprised mainly of white men, and the study was conducted in only one region of the United States.

\section{Clinical bottom line}

As compared to usual care, a multisite, RCT of the CASA collaborative symptom care intervention did not demonstrate improved heart failure-specific health status; however, distressing symptoms related to chronic heart failure, such as depression and fatigue, did improve.

\section{A Focus on Caregivers}

\section{Summary and main findings: Impact of location of death on health care spending of bereaved spouses}

Caring for loved ones at the end of life may impact the health and well-being of the family caregiver. Deaths 
involving intensive, life-prolonging treatments at the end of life have been shown to increase reported post-traumatic stress and to negatively affect the physical and mental health for the caregivers. Research shows that this can lead to increased hospitalizations and overall health care utilization for the bereaved caregiver because they stop participating in preventative health behaviors. ${ }^{24}$ Understanding the effects of end-of-life care on family caregivers needs to be explored to inform care models that help prevent negative health outcomes for caregivers. Research has shown that low intensity end-of-life care, such as hospice and palliative care services provided in the home setting, mitigates the negative effect of caregiving by decreasing depression, and increasing quality of death and adjustment to death of the loved one. ${ }^{25}$ In the article, Impact of In-Hospital death on Spending for Bereaved Spouses, the authors sought to examine how patients' location of death relates to health care utilization and spending for surviving spouses. The study was an observational study. The bereaved spouses whose spouses died in a hospital were matched to those whose spouses died outside the hospital using propensity scores based on decedent and spouse demographic and clinical characteristics, care preferences, and regional practice patterns. The primary outcome was the total Medicare expenditures of the bereaved spouses of decedents who died in the hospital spent $\$ 3,106$ more Medicare dollars $(p=0.04)$ in the first 6 months than those whose spouses did not die in a hospital, and the increased spending continued at 12 months post death $(p=0.02)$. The secondary outcomes were the number of hospitalizations and emergency department visits measured at 12 months after spouse's death. The spouses of those who died in the hospital experienced significantly more emergency department visits $(p=<0.01)$ and hospital admissions $(p=0.02)$. The outcomes revealed an association between location of death and the surviving spouse's subsequent health care utilization.

\section{Strengths and limitations}

The study was a longitudinal observational study that utilized a large nationally represented sample. The limitations to consider are that it was limited to fee for services Medicare services, and that the treatment preferences of the bereaved spouses were unknown.

\section{Clinical bottom line}

Bereaved spouses whose partners died in the hospital had higher Medicare spending and more hospitalization and emergency department visits at 12 months, and these factors persisted at 24 months after their spouse's death. The findings suggest that better support for the needs of caregiving family members may improve their health outcomes and lower downstream health care costs.

\section{Disclaimer}

The content is solely the responsibility of the authors and does not necessarily represent the official views of the National Institutes of Health.

\section{Funding Information}

H.C. is supported by the NIH/NINR (1K99NR0016686). C.B.S. is supported by the American Cancer Society (MRSG-
13-232-01-PCSM). J.N.D.-O. is supported by the NIH/NINR (R00NR015903), the NIH/NCI (R01CA229197), and the National Palliative Care Research Center. L.P.G. is supported by the National Institute on Aging (NIA) (K23AG049930).

\section{Authors Disclosure Statement}

No competing financial interests exist.

\section{References}

1. Allen LA, McIlvennan CK, Thompson JS, et al.: Effectiveness of an intervention supporting shared decision making for destination therapy left ventricular assist device: The DECIDE-LVAD Randomized Clinical Trial. JAMA Intern Med 2018;178:520-529.

2. Fried TR: Shared decision making-finding the sweet spot. N Engl J Med 2016;374:104-106.

3. Stacey D, Légaré F, Lewis KB: Patient decision aids to engage adults in treatment or screening decisions. JAMA 2017;318:657-658.

4. Curtis JR, Wenrich MD, Carline JD, et al.: Understanding physicians' skills at providing end-of-life care. J Gen Intern Med 2001;16:41-49.

5. Curtis JR, Downey L, Back AL, et al.: Effect of a patient and clinician communication-priming intervention on patientreported goals-of-care discussions between patients with serious illness and clinicians: A randomized clinical trial. JAMA Intern Med 2018;178:930.

6. Au DH, Udris EM, Engelberg RA, et al.: A randomized trial to improve communication about end-of-life care among patients with COPD. Chest 2012;141:726-735.

7. Patel MI, Sundaram V, Desai M, et al.: Effect of a lay health worker intervention on goals-of-care documentation and on health care use, costs, and satisfaction among patients with cancer: A randomized clinical trial. JAMA Oncol 2018;4:1359.

8. Kangovi S, Grande D, Trinh-Shevrin C: From rhetoric to reality-community health workers in post-reform U.S. health care. N Engl J Med 2015;372:2277-2279.

9. Patel MI, Periyakoil VS, Moore D, et al.: Delivering endof-life cancer care: Perspectives of providers. Am J Hosp Palliat Care 2018;35:497-504.

10. Patel MI, Periyakoil VS, Blayney DW, et al.: Redesigning cancer care delivery: Views from patients and caregivers. J Oncol Pract 2017;13:e291.

11. Fischer SM, Cervantes L, Fink RM, Kutner JS: Apoyo con Cariño: A pilot randomized controlled trial of a patient navigator intervention to improve palliative care outcomes for Latinos with serious illness. J Pain Symptom Manage 2015;49:657-665.

12. Rosenberg AR, Bradford MC, McCauley E, et al.: Promoting resilience in adolescents and young adults with cancer: Results from the PRISM randomized controlled trial. Cancer 2018;124:3909-3917.

13. Carver CS: Enhancing adaptation during treatment and the role of individual differences. Cancer 2005;104(S11):26022607.

14. Andersen BL, Yang HC, Farrar WB, et al.: Psychologic intervention improves survival for breast cancer patients. Cancer 2008;113:3450-3458.

15. Andersen BL, Thornton LM, Shapiro CL, et al.: Biobehavioral, immune, and health benefits following recurrence for psychological intervention participants. Clin Cancer Res 2010;16:3270. 
16. Andersen BL, Goyal NG, Westbrook TD, et al.: Trajectories of stress, depressive symptoms, and immunity in cancer survivors: Diagnosis to 5 years. Clin Cancer Res 2017;23:52.

17. Sutherland A, Naessens K, Plugge E, et al.: Olanzapine for the prevention and treatment of cancer-related nausea and vomiting in adults. Cochrane Database Syst Rev 2018;9: CD012555.

18. Bekelman DB, Allen LA, McBryde CF, et al.: Effect of a collaborative care intervention vs usual care on health status of patients with chronic heart failure: The CASA randomized clinical trial. JAMA Intern Med 2018;178:511-519.

19. Graham J: IOM report calls for transformation of end-oflife care. JAMA 2014;312:1845-1847.

20. Rogers JG, Patel CB, Mentz RJ, et al.: Palliative care in heart failure: The PAL-HF randomized, controlled clinical trial. J Am Coll Cardiol 2017;70:331-341.

21. Brännström M, Boman K: Effects of person-centred and integrated chronic heart failure and palliative home care. PREFER: A randomized controlled study. Eur J Heart Failure 2014;16:1142-1151.

22. Wong FKY, Ng AYM, Lee PH, et al.: Effects of a transitional palliative care model on patients with end-stage heart failure: A randomised controlled trial. Heart 2016;102: 1100-1108.

23. Heidenreich PA, Spertus JA, Jones PG, et al.: Health status identifies heart failure outpatients at risk for hospitalization or death. J Am Coll Cardiol 2006;47:752-756.

24. Reeves K, Bacon K, Fredman L: Caregiving associated with selected cancer risk behaviors and screening utilization among women: Cross-sectional results of the 2009 BRFSS. BMC Public Health 2012;12:685.

25. Ornstein KA, Garrido MM, Siu AL, et al.: Impact of inhospital death on spending for bereaved spouses. Health Services Res 2018;53 Suppl 1:2696.

Address correspondence to: Heather Coats, PhD, APRN-BC College of Nursing University of Colorado Denver Anschutz Medical Campus Mail Stop C288-18, Education 2 North 13120 E. 19th Avenue Aurora, CO 80045

E-mail: heather.coats@ucdenver.edu 